\title{
Development and Validation of a Porous Theory of Mind Scale
}

\author{
Michiel van Elk \\ Department of Psychology, University of Amsterdam, Amsterdam, the \\ Netherlands \\ m.vanelk@uva.nl \\ David Maij \\ Department of Psychology, University of Amsterdam, Amsterdam, the \\ Netherlands \\ D.L.R.Maij@uva.nl \\ Bastiaan Rutjens \\ Department of Psychology, University of Amsterdam, Amsterdam, the \\ Netherlands \\ bastiaan.rutjens@gmail.com
}

\begin{abstract}
We report the results of an empirical investigation of the extent to which supernatural believers endorse a porous conception of the mind, i.e., the belief that one's thoughts can be directly perceived by others. We developed a porous theory of mind (PToM) scale, tested its factor structure by using both exploratory and confirmatory factor analyses, and showed its relation with supernatural beliefs in three studies in the Netherlands and one study with North-American participants. We found that endorsement of a PToM is positively related to paranormal beliefs. We also showed that the endorsement of a porous view of the mind differs in meaningful ways between people from different religious backgrounds (i.e., Protestants vs. Catholics; spiritualists vs. religious believers). The finding that supernatural believers endorse a porous conception of the mind fits well with recent work in the field of the anthropology of religion and the psychology of paranormal beliefs. We propose that our PToM scale provides a parsimonious measurement tool (i.e., consisting only of 4 items) that circumvents socially desirable responding, while providing direct insight in the endorsement of paranormal beliefs.
\end{abstract}




\section{Keywords}

supernatural beliefs - theory of mind - porous self - paranormal beliefs - religiosity

\section{Introduction}

Central to many religions is the notion that God has access to our deepest thoughts and feelings (Haslam, Kashima, Loughnan, Shi, \& Suitner, 2008), as exemplified for instance by the following passage from the biblical book of Psalms, considered by Jews and Christians as a part of their Scriptures: 'Search me, O God, and know my heart: try me, and know my thoughts' (Psalm 139:23; KJV). Moreover, many believers are convinced that supernatural entities, like God or Satan, can place thoughts in our mind as well (Irmak, 2014). In fact, the ability to distinguish one's own thoughts from externally inserted thoughts is key to many new religious and spiritual movements, such as charismatic Pentecostal churches and a variety of New Age and psychic beliefs. For instance, field studies in the Vineyard Pentecostal church have shown that believers learn to hear the voice of God through a process referred to as 'inner sense cultivation' (Luhrmann, 2012). Basically, by paying careful attention to one's inner thoughts and sensations believers gradually learn to attribute spontaneous thoughts to God. Many psychics are convinced that they have a special capability for mind-reading (Fenwick, Galliano, Coate, Rippere, \& Brown, 1985) or for communicating with spirits of deceased ancestors (Winkelman, 2002). Within New Age communities different courses and training programs on the development of psychic abilities can be found, which often share the notion that one should pay close attention to one's inner sensations and learn to reduce one's natural tendency to inhibit or discredit spontaneous thoughts and images (Luhrmann, 1991).

The fact that believers in supernatural entities frequently report the experience of externally attributed thoughts, implies that believers may endorse a different concept of the human mind than skeptics and non-believers. It has been suggested that the prototypical Western conception of the mind is best captured by the 'Mind as container'-metaphor, i.e., the mind is a container filled with private thoughts and feelings to which only the person him/herself has access (Gibbs \& Obrien, 1990; Lakoff \& Johnson, 1980). It has also been suggested that the Western notion of the mind is 'bounded', reflecting the assumption that outside entities cannot enter the mind and that inner thoughts cannot leave the mind (Taylor, 2007). In contrast, non-western cultures and many religions may be characterized by a 'porous' view of the mind, according 
to which supernatural entities can enter the mind (Lillard, 1998). Endorsing a 'porous theory of mind' (PToM) implies that direct communication between different minds is possible: from human minds to supernatural entities (e.g., as observed in prayer) and from supernatural entities to human minds (e.g., as observed in supernatural revelations, or when hearing God's voice). Thus, a central feature of many supernatural beliefs may be the endorsement of a porous conception of the mind (Cassaniti \& Luhrmann, 2011).

Surprisingly, although many cross-cultural and ethnographical studies have shown cultural variations in theory of mind - ascribing different functions and importance to the concept of 'mind' (Lillard, 1998; Riekki, Lindeman, \& Lipsanen, 2013) - the idea that different groups of believers in supernatural phenomena within Western cultures may be characterized by endorsing different gradations of a PToM has hardly been investigated. Here, we hypothesized that we should observe meaningful differences in the conception of the mind as being bounded vs. porous, depending on one's religious and spiritual beliefs. That is, we hypothesized that a porous conception of the mind is at the heart of many spiritual and paranormal beliefs (Irwin, 2009), as well as of many evangelical beliefs (Luhrmann, 2012) that are characterized by the notion that mind-reading and communication with supernatural entities is possible. In contrast, religious communities that actively discourage the belief that direct personal communication between God and men is possible, such as Orthodox Protestant believers, should be characterized by a more bounded view of the human mind (Emmons \& Sobal, 1981; Rice, 2003).

To investigate our hypotheses, we developed a novel scale to measure the extent to which people view the human mind as bounded vs. porous. Across four studies involving paranormal believers, religious believers and non-believers from different denominations in both the Netherlands and the US we investigated the relation between our PToM scale with paranormal and traditional religious beliefs.

As a starting point for constructing a new scale, we built on the notion that linguistic metaphors often reflect the implicit concepts that are prevalent in one's culture (Gibbs \& Obrien, 199o; Lakoff \& Johnson, 1980). These metaphors are typically derived by applying properties from a concrete physical domain to an abstract target domain (e.g., 'importance = weight'; 'love = warmth' etc.). We thus created variants of the 'mind-as-container' metaphor by presenting participants with different metaphors describing the mind as reflecting either a transparent or a porous entity (e.g., 'The mind is like a glass cube'; 'The mind works like an antenna'). We asked participants to what extent they thought each metaphor was an accurate description of the human mind. Next, the different items were combined in a PToM scale. We investigated the relation 
between the PToM scale and paranormal and traditional religious beliefs by including well-established scales to measure these beliefs (Jong, Bluemke, \& Halberstadt, 2013; Tobacyk, 2004). The first two studies were partly conducted at psychic fairs in order to obtain sufficient variability in religious and paranormal beliefs (for similar approach, see: van Elk, 2013; 2015). The third study was conducted in the Netherlands, targeting specific groups of orthodox Dutch Protestant and Catholic believers (see also: van Elk, Rutjens, \& van Harreveld, 2017). The fourth study was conducted by recruiting a large sample of participants from Amazon's Mechanical Turk system living in North-America (Buhrmester, Kwang, \& Gosling, 2011).

\section{$2 \quad$ Study 1}

\subsection{Methods}

\subsubsection{Participants}

The first study was conducted as a field study at a psychic fair in the Netherlands (Paranormaal Alternatief, September 2014, Rijswijk, the Netherlands). Visitors of the fair were invited to participate in a study on the 'psychological basis of paranormal beliefs', by completing a computerized or a paper-and-pencil version of a questionnaire. All participants gave informed consent before participating and were offered a fair-trade chocolate bar upon completing the questionnaire. In the first study, in total 71 respondents participated (14 males; mean age $=44.67$ years, $\mathrm{SD}=14.66$; age range $=19-68$ years ).

\subsubsection{Materials and Analysis}

Participants were presented with a questionnaire, measuring basic demographic variables (age, gender, level of education), traditional religious beliefs (using the 'supernatural belief scale'; sBs; 10 items; Cronbach's $\alpha=.86$; cf. Jong et al., 2013) and paranormal beliefs (using a shortened version of the revised paranormal belief scale; RPBS; 15 items; Cronbach's $\alpha=.91$; Tobacyk, 2004). The SBS scale was administered using an 8-point Likert scale ( 1 = strongly disagree to $8=$ strongly agree), the RPBS scale was administered as a 7-point Likert scale $(1=$ strongly disagree to $7=$ strongly agree $)$ and for each subject the mean scale score was calculated.

To measure belief in a porous theory of mind (PToM), first participants were presented with the following instruction (translated from Dutch): 'In different cultures, different metaphors have been used to describe the mind. Below, different metaphors are presented. Please indicate to what extent you believe that the metaphor describes the human mind'. The following metaphors were included: 
The human mind is like a glass cube; others can read your thoughts as well. The human mind works like an antenna; signals from the outside may be received and sent.

The human mind is like a treasure box; you have the key, but everyone who is capable to open the lock has access to your thoughts and feelings.

The human mind is like an Internet router, receiving and sending thoughts and feelings.

Participants indicated to what extent they agreed or disagreed for each statement on a 7 -point Likert scale $(1=$ strongly disagree; 7 = strongly agree $) .{ }^{1}$

A factor analysis was conducted to determine the underlying factors of the PToM items. Following (Russell, 2002), a principal axis factoring (PAF) analysis without rotation was performed on the 4 item scores. Based on visual inspection of the scree-plot a solution with one underlying factor was selected. This solution was confirmed by using parallel analysis in which the observed eigenvalues were compared to the distribution of eigenvalues obtained through 500 random permutations (Glorfeld, 1995). To investigate whether the PToM scale was associated with supernatural and paranormal beliefs, we conducted a correlation analysis in which we correlated the mean PToM score with the other variables (i.e., age, education, religiosity and paranormal beliefs).

\subsection{Results \\ 2.2.1 Descriptive Statistics}

The average score on the RPBS was $5.03(S D=1.09$; range $=2.80-7.00)$, reflecting that overall our participants strongly believed in paranormal phenomena (range: $1=$ strongly disagree; $7=$ strongly agree). The average score on the SBS was $4.81(S D=1.51$; range $=1.30-8.00)$, indicating a moderate to strong belief in traditional religious concepts (range: $1=$ strongly disagree; $8=$ strongly agree). The average score on the PToM scale was $4.54(S D=1.42$; range $=1.00-7.00)$, reflecting that participants tended to agree that the porous theory of mind metaphors were an accurate reflection of the human mind (range: $1=$ strongly disagree; 7 = strongly agree).

1 Initially, we also included 3 additional items from the magical ideation scale that were related to mind-reading (Eckblad \& Chapman, 1983) and a reverse-coded metaphor (representative of a bounded conception of the human mind). However, because these items were conceptually different from the other PToM items and as previous studies already showed a relation between magical ideation and paranormal beliefs (Thalbourne \& French, 1995; Williams \& Irwin, 1991) we did not include these items in the final analyses. 
TABLE 1 Porous Theory of Mind items in Study 1 and factor loadings based on the principal factoring analysis

Factor 1

The human mind is like a glass cube; next to you, others can read thoughts $\quad .54$ as well.

The human mind works like an antenna; signals from the outside may be

.84 received and sent.

The human mind is like a treasure box; you have the key, but everyone

capable of opening the lock has access to your thoughts and feelings.

The human mind is like an internet router, receiving and sending thoughts

.81 and feelings.

\subsubsection{Principal Axis Factoring Analysis of the PToM scale}

The Kaiser-Meyer-Olkin measure verified the sampling adequacy for the factor analysis; KMO = .76 (Field, 2009). Bartlett's test of sphericity; $\chi^{2}(6)=96.26$, $p<.001$, indicated that the correlations between the items were appropriate for doing a PAF analysis. An initial analysis was run in which eigenvalues were obtained for each of the components. Based on visual inspection of the plot of Cattell's scree test, a one-factor solution was selected, accounting for $53.1 \%$ of the variance (see Table 1 for items and factor loadings). This analysis was confirmed by using parallel factor analysis (Glorfeld, 1995), indicating that only the eigenvalue of a 1-factor solution fell outside the 95th percentile of the randomization distribution. Accordingly, the 4 PToM items were combined in an average scale score, with a reliability of $\alpha=.8$.

\subsubsection{Correlation Analysis}

As can be seen in Table 2, the PToM scale correlated strongly with traditional religious beliefs (as measured with the sBs) and with paranormal beliefs (as measured with the RPBS). Age was positively associated with religious and paranormal beliefs, but no significant relationship between education and religious and paranormal beliefs was observed.

\subsection{Discussion}

In the first study we established that the PToM items loaded on one factor (using Exploratory Factor Analysis; the one-factor structure was confirmed using Confirmatory Factor Analysis in Study 4), reflecting the extent to which people endorse a porous conception of the mind. We found that a stronger 
TABLE 2 Pearson's correlations in Study 1 between Age, Education, the Porous Theory of Mind scale (PToM), the supernatural belief scale (sBs) and the revised paranormal belief scale (RPBS)

\begin{tabular}{|c|c|c|c|c|c|}
\hline & Age & Edu & PToM & SBS & RPBS \\
\hline Age & - & -.09 & -.09 & .32 & $.39^{* *}$ \\
\hline Edu & & - & -.17 & -.10 & -.16 \\
\hline PToM & & & - & $.40^{* * *}$ & $\cdot 5^{1 * * *}$ \\
\hline SBS & & & & - & $.64^{* * * *}$ \\
\hline RPBS & & & & & - \\
\hline
\end{tabular}

Note: ${ }^{*} p<.05,{ }^{* *} p<.01,{ }^{* * *} p<.001$

endorsement of a porous notion of the mind was positively related to traditional religious beliefs and even more strongly to paranormal beliefs. These findings are in line with the theoretical suggestion that supernatural believers are characterized by a different conception of 'mind' than skeptics and nonbelievers (Cassaniti \& Luhrmann, 2011).

An obvious concern in the first study is that the relation between the PToM scale and paranormal or religious beliefs can actually be explained by a conceptual overlap in the items used to measure PToM (e.g., 'The human mind works like an antenna; signals from the outside may be received and sent.') and paranormal beliefs (i.e., 'Mind reading is possible'). This problem is also known as the common method bias, in which the variance in the dependent measures is to some extent caused by the use of the same measurement tool (i.e., self-report questionnaires; cf. Podsakoff, MacKenzie, Lee, \& Podsakoff, 2003). However, when looking separately at the inter-item correlations, we found that the PToM scale was significantly related to all individual RPBS items (except for belief in astrology), many of which did not have an obvious relation with 'mindreading' (e.g., psychokinesis; predicting the future etc.). In addition we found that the PToM scale was positively related to traditional religious beliefs, which did not have any association with psychic abilities.

A second concern was that in Study 1 participants were recruited at a psychic fair and this likely resulted in a selective and potentially biased sample. Accordingly, in Study 2 we aimed to replicate and extend the findings to a broader population. Therefore, in Study 2 we recruited visitors of a psychic fair, but also visitors of a Christmas fair to participate in our study. This way we aimed to obtain more variability in our measures of religious and paranormal beliefs. In addition, by testing participants at a psychic fair and a Christmas 
fair we could also establish the construct validity of the PToM scale, as we expected that psychic believers (visiting the psychic fair) should endorse a more porous view of the mind than people who did not explicitly believe in psychic phenomena (i.e., participants visiting the Christmas fair).

\section{$3 \quad$ Study 2}

\subsection{Methods}

3.1.1 Participants

The second experiment was conducted as a combined field and online study. Visitors of a psychic fair (Paraview, Heemstede, the Netherlands, December 2014) were invited to participate in a study on the 'psychological basis of paranormal beliefs' by completing a computerized questionnaire. They were offered a fair-trade chocolate bar upon completing the questionnaire. Visitors of a Christmas fair (Kerstfair, De Broodfabriek, Rijswijk, December 2014) were also invited to participate and received a voucher with a link to the same online study. Participants at the Christmas fair were offered a small fairtrade chocolate bar in advance and could win a lottery (4 times a voucher of 25 Euros) upon completing the questionnaire at home. All participants gave informed consent before participating in the study. In total 150 respondents participated (40 males; mean age $=44.8$ years, $S D=15.3$; age range $=$ $14-81$ years).

\subsubsection{Materials and Analysis}

Similar to the first study, participants indicated their age, gender and level of education and their paranormal beliefs (RPBS; 15 items; Cronbach's $\alpha=.96$ ). Belief in a Porous Theory of Mind was measured using the same 4 items as used in Study 1 (Cronbach's $\alpha=.82$ ). Traditional religious beliefs were assessed using four items ('To what extent do you consider yourself religious?'; 'To what extent do you believe in God?'; 'How often do you visit a church?'; 'How often do you pray?'; Cronbach's $\alpha=.82$ ).

The descriptive statistics and the correlations between the different scales are reported. We also compared the scores on the different scales between the two different groups that we tested (i.e., participants tested at the psychic fair vs. the Christmas fair) to establish the construct validity of our measure; we expected that visitors of the psychic fair would view the mind as being more porous than visitors of the Christmas fair. 


\subsection{Results \\ 3.2.1 Descriptive Statistics}

The average score on the RPBS was $3.94(S D=1.56$; range $=1.00-7.00)$, reflecting that overall our participants believed moderately in the existence of paranormal phenomena (range: $1=$ strongly disagree; $7=$ strongly agree). The average score on the scale measuring traditional religiosity was 2.58 $(S D=1.41$; range $=$ 1.00-7.00), indicating a relatively weak belief in traditional religious concepts (range: 1 = strongly disagree; 7 = strongly agree). The average score on the PToM scale was $4.14(S D=1.48$; range $=1.00-7.00)$, reflecting that overall participants tended to endorse a porous conception of the mind (range: $1=$ strongly disagree; 7 = strongly agree).

When directly comparing the data collected in the different contexts, we found that participants at the psychic fair scored higher on the PToM scale than participants at the Christmas fair, $F(1,148)=16.60, p<$.oo1 (see Table 3 ). The groups did not differ in traditional religious beliefs $(F(1,148)<1)$, but participants at the psychic fair scored higher on paranormal beliefs than participants at the Christmas fair, $F(1,148)=49.69, p<.001$.

\subsubsection{Correlation Analysis}

The correlations between the different variables are presented in Table 4. As can be seen, significant positive correlations were observed between the PToM scale, traditional religious beliefs and the RPBS. Furthermore, Education was negatively related to the PToM scale and paranormal beliefs.

\subsection{Discussion}

The second study extended the notion that endorsing a porous notion of the mind is related to paranormal beliefs, by using a larger and religiously more diverse group than in Study 1. In addition, we established the construct validity of the PToM scale, by showing that a group of people recruited at a psychic

TABLE 3 Differences between groups on traditional religious beliefs (TRB), paranormal beliefs (RPBS) and porous theory of mind (PToM). ** $\mathrm{p}<.001$

\begin{tabular}{llll}
\hline & TRB & RPBS & PToM \\
& & & \\
\hline Group 1: Psychic Fair & $2.65(1.41)$ & $4.71(1.27)$ & $4.61(1.36)$ \\
Group 2: Christmas Fair & $2.52(1.43)$ & $3.16(1.43)$ & $3.67(1.45)$ \\
\hline
\end{tabular}


TABLE 4 Correlations in Study 2 between Age, Education, the Porous Theory of Mind scale (PToM), traditional religious beliefs (TRB) and the revised paranormal belief scale (RPBS)

\begin{tabular}{|c|c|c|c|c|c|}
\hline & Age & Edu & PToM & TRB & RPBS \\
\hline Age & - & .036 & -.14 & .06 & .00 \\
\hline Edu & & - & $-.22 * *$ & .010 & $-.35^{* *}$ \\
\hline PToM & & & - & $.26^{* *}$ & $.70^{* * *}$ \\
\hline TRB & & & & - & $34^{* * * *}$ \\
\hline RPBS & & & & & - \\
\hline
\end{tabular}

Note. ${ }^{*} p<.05,{ }^{* *} p<.01,{ }^{* * *} p<.001$.

fair endorsed a more porous view of the mind than participants recruited at a Christmas fair. Thus the concept of a porous view of the human mind may be a central feature of belief in psychic phenomena. The elegance of the PToM scale lies in the fact that by simply asking people about their view of the human mind as being bounded vs. porous using different metaphors, we obtain a measure that has a high predictive validity for establishing whether people believe in paranormal phenomena and to what extent they believe in supernatural phenomena. A potential advantage of using this scale is that it avoids socially desirable responding, as most people probably won't be aware of the relation between the PToM scale and belief in paranormal and religious phenomena.

In both Study 1 and 2 we found that the PToM scale was more strongly related to paranormal beliefs than to religious beliefs. This may be related to the fact that in both studies participants were recruited at a psychic and a Christmas fair, and participants may have strongly endorsed psychic but not necessarily religious beliefs. However, some mainstream religions (e.g., orthodox Protestantism) actually reject the possibility of 'mind reading' or consider the belief that spirits and entities crossing the boundaries of the human mind occult (Boyd, 1996; Emmons \& Sobal, 1981; Rice, 2003). Therefore, some religious believers will likely view the mind as being bounded rather than porous - which could explain why the PToM scale primarily predicts psychic but to a lesser extent traditional religious beliefs in the first two studies. In contrast, other groups of religious believers may actually agree with the view that the human mind is porous, as anthropological studies have shown for instance that Pentecostal religious denominations may be characterized by endorsing a porous conception of the mind (Cassaniti \& Luhrmann, 2011; Luhrmann \& 
Morgain, 2012; Luhrmann, Nusbaum, \& Thisted, 2010). Specifically, Evangelical Christians in the US view the mind as a porous entity, which in turn enables communication with God and experiences of hearing God's voice.

Thus, we should expect meaningful differences between traditional religious believers from different denominations in their view of the human mind. Accordingly, we tested whether different groups of believers would respond differently to the PToM scale that we developed. We tested for differences in the endorsement of a porous vs. a bounded view of the human mind in the Netherlands (by comparing orthodox Protestant and Catholic believers; Study 3) and in the US (by comparing self-identified religious, spiritual or atheist participants; Study 4).

In Study 3 we define Orthodox as those participants endorsing strong beliefs in God. This criterion was operationalized by using a selection criterion: participants were only offered the possibility to complete the survey if they rated their religiousness and belief in God with a 4 or higher, with the scale ranging from 1 (not at all) to 5 (very much; for details on procedure, see: van Elk et al., 2017). The rationale for comparing orthodox Protestant and Catholic believers was that in this way we could establish whether two groups of traditional religious believers (who both strongly believe in traditional religious teachings and concepts) differ in their views of the human mind. Such a finding would entail that within different religious communities, local differences exist in people's view of mind, which are shaped by one's cultural upbringing and religious training (Cassaniti \& Luhrmann, 2014).

In Orthodox Protestantism (i.e., the Dutch reformed church), personal experiences of the divine (e.g., hearing God's voice, seeing visions) are often discouraged (Schmidt, 2000), and a strong emphasis is placed instead on revelation through the Holy Scripture alone (i.e., sola scriptura). The idea that God can communicate with humans by placing 'thoughts in their minds' is actually viewed as being reminiscent of psychic beliefs and is disapproved by the official teachings of the church (Boyd, 1996; Emmons \& Sobal, 1981; Rice, 2003). Mainstream Catholicism also does not actively encourage personal revelatory experiences of God, but neither does it explicitly reject the view that mindreading is possible. Catholics are also less individualistic than Protestants (Cohen, Hall, Koenig, \& Meador, 2005) and tend to make more external dispositional attributions (Li et al., 2012) compared to Protestants. Accordingly, based on these denominational differences we expected that Protestants would score low on our PToM scale, reflecting a bounded conception of the mind, whereas Catholics were expected to score at intermediate levels, reflecting a somewhat more porous and interdependent view of the mind. 


\section{Study 3: Catholics vs. Protestants}

\subsection{Methods}

4.1.1 Participants

The third experiment was conducted in collaboration with a Dutch research agency (i.e., Motivaction), which has access to a representative sample of Dutch participants (>100.00o respondents). In total 404 participants (188 men) completed our study; their mean age was 54.4 years (range $=18-70, S D=12.6$ ). Participants received credits which they could use to buy presents or gift vouchers in an online shop. We were specifically interested in recruiting highly religious Protestant and Catholic participants and part of this data was used for a different study on prosocial behavior (van Elk et al., 2017).

\subsubsection{Materials and Analysis}

Similar to the first study, participants indicated their age, gender and level of education. Traditional religious beliefs were assessed using six items ('To what extent do you consider yourself religious?'; 'To what extent do you believe in God?', 'How often do you visit a church?'; 'How often do you pray?'; 'My faith is important to me.'; 'My faith affects my daily practices and thinking.; Cronbach's $\alpha=.89$ ). The descriptive statistics and the correlations between the different scales are reported.

\subsection{Results \\ 4.2.1 Descriptive Statistics}

The average score on the scale measuring traditional religiosity was 4.08 $\left(S D=.70\right.$; range $\left.=2.5^{\circ}-5.00\right)$, indicating that our participants strongly endorsed religious beliefs (range: $1=$ strongly disagree; $5=$ strongly agree). The average score on the PToM scale was 3.80 $(S D=1.20$; range $=1.00-7.00$; range: $1=$ strongly disagree; 7 = strongly agree). Protestants scored higher on religious beliefs $(M=4.41, S D=.61)$ than Catholics $(M=3.76, S D=.64), t(402)=10.5$ o, $p<.001$. In contrast, Protestants scored lower on the PToM scale $(M=3.42, S D=1.23)$ than Catholics $(M=4.17, S D=1.06), t(402)=-6.6 \circ, p<.001$.

\subsubsection{Correlation Analysis}

The correlations between the different variables are presented in Table 5. As can be seen, a significant negative correlation was observed between the PToM scale and traditional religious beliefs. However, when looking at the withingroup correlations we found that for Protestants the PToM score was negatively correlated to religious beliefs, $r=-.26, p<.001$, whereas for Catholics the PToM Score was positively correlated to religious beliefs, $r=.14, p<.05$. 
TABLE 5 Correlations in Study 3 between Age, Education, the Porous Theory of Mind scale (PToM) and traditional religious beliefs (ТRв). ${ }^{*} \mathrm{p}<.05,{ }^{* *} \mathrm{p}<.001$

\begin{tabular}{lllll}
\hline & Age & Edu & PToM & TRB \\
\hline Age & - & $.20^{* *}$ & .05 & $-.11^{*}$ \\
Edu & & - & -.05 & $<-.01$ \\
PToM & & & - & $-.20^{* *}$ \\
TRB & & & & - \\
\hline
\end{tabular}

\subsection{Discussion}

In the third study, we found that traditional Catholic believers scored higher on our PToM scale than orthodox Protestant believers. In addition, we found that among Protestants viewing the mind as porous (vs. bounded) was negatively related to traditional religious beliefs, whereas among Catholic participants viewing the mind as porous (vs. bound) was positively related to religious beliefs. These findings indicate that whereas the view that the mind is porous may be a distinctive feature of some religious communities (e.g., Evangelicals; Catholics), it is not a universal characteristic of religious communities in general. We propose that the endorsement of a porous view of the human mind is specifically related to frequent and intense experiences of the supernatural, when these are actively encouraged within one's community (Luhrmann, 2012). Indeed, in a prospective study in the US we found that higher scores on our PToM scale were related to more vivid experiences of God and more frequent daily spiritual experiences (Luhrmann et al., in prep.).

All studies reported in this paper were conducted in the Netherlands. In order to provide a validation of our PToM measure in a different country, in Study 4 we recruited a large sample of participants from North America. In order to obtain sufficient variability in our religiosity and paranormal belief measures, we also used screening questions asking whether participants considered themselves a Christian, spiritual believer or a non-believer / atheist. By using a stratified selection procedure, we were able to recruit approximately the same number of self-identified Christians, spiritualists and atheists, thereby providing an additional opportunity to establish the construct validity of our PToM scale. That is, we expected that self-identified spiritualists would score highest on our PToM, followed by Christians and next by atheists, indicating that different groups of self-identified believers or non-believers can be characterized by a different view of the human mind. 
An additional aim of Study 4 was to more firmly establish the factor structure of our PToM scale, by using a confirmatory analysis (Gerbing \& Hamilton, 1996), instead of the exploratory factor analysis that was used in Study 1. By determining the relation between our English version of the PToM scale and religious and paranormal beliefs, we could further establish the construct validity of our scale.

\section{5}

\section{Study 4}

$5 \cdot 1$

\section{Methods}

5.1.1 Participants

The fourth study was conducted as an online study using Amazon's Mechanical Turk participant panel and was part of a larger research project in which different additional measures were included (Maij et al., 2017). The study was advertised among North-American MT workers, as a study on the relation between religion and behavior. In order to obtain sufficient variability in religious and spiritual beliefs, participants were first asked whether they considered themselves 'Christian,' 'Muslim,' 'Hindu', 'spiritual', 'atheist' or otherwise. By using a selection procedure care was taken that we created groups of Christians, spiritual believers and atheists of approximately the same size. In total, after exclusion of participants with missing responses, data from 772 MT workers was included in the analysis ( 361 females; $M$ age $=34.9$ years, $S D=11.0$; range = 18-71). Participants were offered a financial remuneration for their participation through Amazon's MT system.

\subsubsection{Methods \& Analysis}

Similar to the second study participants indicated their age, gender and level of education, their paranormal beliefs (RPBS; 15 items; Cronbach's $\alpha=.96$ ). Traditional religious beliefs were assessed using three items ('To what extent do you believe in God?'; 'How often do you visit a church?'; 'How often do you pray?'; Cronbach's $\alpha=.85$ ).

\subsubsection{Psychometric Scale Properties}

The same four PToM items were used as in the first three studies (Cronbach's $\alpha=.70 ; 1=$ 'I don't agree at all'; $5=$ 'I very much agree'). A confirmatory factor analysis (CFA) was performed using lavaan (Rosseel, 2012), an add-on package for structural equation modeling in the statistical programming environment $R$ $(\mathrm{RC}, 2014)$. Parameters of the CFA model were estimated using the robust maximum likelihood (MLR) estimator and missing data was taken into account by 
means of full information maximum likelihood (FIML) estimation. When data are non-normally distributed, ML standard errors may be biased downwards. The MLR estimator yields the same parameter estimates as ML estimation, but yields corrected standard errors, which are not biased downwards under non-normality (Chou, Bentler, \& Satorra, 1991). In addition, Enders (2001) has shown that the combined use of FIML estimation and robust standard errors can substantially reduce negative impact of non-normally distributed and missing data. The scale of the latent variable was identified by fixing the value of the first factor loading to a value of one.

We will judge model fit based on the values of CFI and SRMR, as well as the estimated parameter values and their standard errors. Although we will also report RMSEA, this measure of fit presents with a positive bias in models with low degrees of freedom, which increases with lower degrees of freedom. In our model, the latent variable is measured by only 4 items, yielding only 2 degrees of freedom. In such a case, a positive bias is to be expected for RMSEA. We therefore assumed SRMR, which represents the standardized difference between observed and model-implied correlations, as well as CFI, which compares the fit of the fitted models to that of a baseline model of independence, to provide more appropriate indicators of model fit in the current study.

The construct validity of the PToM scale was confirmed by using convergent approaches. By using correlation analyses the relation between PToM and traditional religious beliefs and paranormal beliefs was investigated. In addition, by comparing the scale-scores between the different self-identified groups the construct validity was confirmed.

\section{$5.2 \quad$ Results}

\subsubsection{Confirmatory Factor Analysis}

The parameter estimates of the unidimensional model were as hypothesized: All loadings were positive and significantly different from zero. All standardized loadings showed values equal to or greater than .48, indicating a medium to large linear association between each of the items and the factor (see Table 6). The variance of the latent trait was: $.34, \mathrm{SE}=0.04, p=<.001$. According to the model fit indices, the unidimensional model showed acceptable fit to the data: The $\chi^{2}$ was significant, which is to be expected with a sample size of 772 , and should not be taken as an indicator of model misfit $\left(\chi^{2}(2)=\right.$ 53.42; $p<.001)$. The comparative fit index indicated an acceptable fit $($ CFI $=0.93)$, the Standardized Root Mean Squared Residual indicated a good measure of fit (SRMR $=.04)$, whereas the root mean squared error of approximation indicated unacceptable fit $($ RMSEA $=0.14)$. However, 
TABLE 6 Item loadings for the PToM scale as established using confirmatory factor analysis. CSL $=$ Completely Standardized Loadings

\begin{tabular}{llllll}
\hline & Loadings & SE & z-value & $\mathrm{P}(>|\mathrm{z}|)$ & CSL \\
\hline PTOM_1 & 1.00 & 0.58 & & & \\
PTOM_2 & 1.44 & 0.09 & 15.49 & $<.001$ & 0.84 \\
PTOM_3 & 0.94 & 0.07 & 13.99 & $<.001$ & 0.55 \\
PTOM_4 & 1.16 & 0.07 & 16.589 & $<.001$ & 0.67 \\
\hline
\end{tabular}

as the RMSEA may be biased because of the small number of items and the corresponding low degrees of freedom, the CFI and SRMR provide more robust fit indices in the present study and indicate a sufficient fit of our model.

\subsubsection{Descriptive Statistics}

The average score on the RPBS was $2.98(S D=1.43$; range $=1.00-6.94)$, reflecting that overall our participants did not strongly believe in the existence of paranormal phenomena (range: $1=$ strongly disagree; $7=$ strongly agree). The average score on the scale measuring traditional religiosity was $3.25(S D=1.93$; range $=1.00-7.00)$, indicating a relatively weak belief in traditional religious concepts (range: $1=$ strongly disagree; $7=$ strongly agree). The average score on the PToM scale was $2.81(S D=.90$; range $=1.00-5.00 ; 1=$ strongly disagree; $5=$ strongly agree).

As can be seen in Table 7, self-identified spiritual believers scored higher on the PToM scale than religious believers, and self-identified atheists scored lowest on the PToM scale, $F(2,769)=31.01, p<.001, p \eta^{2}=.08$ (all post-hoc

TABLE 7 Scores on the PToM scale, traditional religious beliefs (TRB) and paranormal beliefs (RPBS) for the different self-identified groups of Atheist, religious believers and spiritual believers in Study 4. ${ }^{* * *} p<.001$

\begin{tabular}{llll}
\hline & & & \\
& PToM** & TRB** & RPBS** \\
\hline Atheists & $2.49(.90)$ & $1.38(.64)$ & $2.02(1.15)$ \\
Religious believers & $2.92(.89)$ & $5.20(1.25)$ & $3.28(1.25)$ \\
Spiritual believers & $3.07(.80)$ & $3.23(1.28)$ & $3.74(1.30)$ \\
\hline
\end{tabular}


TABLE 8 Correlations in Study 4 between Age, Education (Edu), the porous theory of mind scale (PToM), traditional religious beliefs (ТRв) and the revised paranormal belief scale (RРвS)

\begin{tabular}{|c|c|c|c|c|c|}
\hline & Age & Edu & PToM & TRB & RPBS \\
\hline Age & - & $.098^{* *}$ & -.039 & $.185^{* * *}$ & $.073^{*}$ \\
\hline Edu & & - & -.06 & -0.02 & $-0.123^{* *}$ \\
\hline PToM & & & - & $0.197^{* *}$ & $0.396 * *$ \\
\hline TRB & & & & - & $0.385^{* * *}$ \\
\hline RPBS & & & & & - \\
\hline
\end{tabular}

Note. ${ }^{*} p<.05,{ }^{* *} p<.01,{ }^{* * *} p<.001$

contrasts were significant, $p<.05$, indicating that the groups differed on the scores on the PToM scale). The groups also differed in expected directions in their general religiosity, $F(2,769)=829.47, p<.001, p \eta^{2}=.68$ (all posthoc contrasts were significant, $p<.001)$ and in their beliefs in paranormal phenomena, $F(1,769)=135.59, p<.001, p \eta^{2}=.26$ (all post-hoc contrasts were significant, $p<.001)$

\subsubsection{Correlation Analysis}

In a correlation analysis, the relation between the PToM scale and the demographic and religious and paranormal variables was investigated. As can be seen in Table 8, the PToM scale was positively related to religious and paranormal beliefs. When looking at the within-group correlations we found that the PToM scale was positively related to paranormal beliefs in all sub-groups (atheists: $r=.31, p<.001$; religious believers: $r=.36, p<.001$; spiritual believers: $r=.25, p<.001$ ). In contrast, the PToM scale was only related to religious beliefs in the self-identified atheist group, $r=.18, p=.003$.

\subsection{Discussion}

In the fourth study, we further validated an English version of the PToM scale in a large sample of participants from the US and Canada. By using a confirmatory factor analysis, we also independently confirmed the one-factor structure of our scale. Similar to the first two studies, we found that the PToM scale was strongly related to paranormal beliefs. As expected, we also observed that different groups of believers differed in meaningful ways in their endorsement of a porous view of the mind: self-identified spiritualists scored highest on the 
PToM scale - reflecting a strongly porous view of the mind - followed by religious believers and next by atheists.

\section{6}

\section{General Discussion}

Across four studies, we developed and validated a novel scale to measure the extent to which believers from different backgrounds and religious communities perceive the human mind to be bounded versus porous (Taylor, 2007). Overall, we found that higher scores on the porous theory of mind scale (PToM) were positively related to paranormal beliefs, but not to traditional religious beliefs. These findings indicate that the perception of the mind as a porous entity may be a defining feature of belief in paranormal phenomena. Paranormal beliefs, such as belief in precognition, telepathy, mindreading and telekinesis all imply the possibility that thoughts can literally enter or exit the mind (Lindeman \& Svedholm, 2012). A main advantage of using the PToM scale as a measurement instrument (i.e., over and beyond classical measures of paranormal beliefs) is that it may elicit less socially desirable responding, because participants may feel more reluctant to agree that they endorse a porous metaphor of the mind, rather than agreeing that paranormal phenomena exist. A related advantage is that the PToM scale is positively related to paranormal beliefs, without evoking associations with supernatural concepts, which could indirectly influence participants' responding (Gervais \& Norenzayan, 2012). We thus propose that the newly developed PToM scale may provide a parsimonious and elegant way to measure and predict belief in paranormal phenomena.

The relation between a porous view of the mind and traditional religious beliefs was more complex and differed between the specific groups that were tested. We found that the PToM scale was positively related to religious beliefs in a group of psychic believers (Study 1 and 2), in a group of orthodox Catholic participants (Study 3) and across a group of self-identified religious believers, spiritualists and atheists (Study 4). However, a negative relation was observed between the PToM scale and religious beliefs for orthodox Protestant participants (Study 3). When looking separately at the self-identified religious believers no association was observed between the PToM scale and religious beliefs (Study 4). These findings show that different groups of religious believers differ in their endorsement of a porous conception of the mind. In some mainstream religions (e.g., orthodox Protestantism) belief in psychic phenomena like 'mindreading' is rejected as being occult (Emmons \& Sobal, 1981; Rice, 2003). In contrast, in other religious denominations (e.g., Evangelicals) belief in and practice of psychic phenomena (e.g., such as hearing the voice of God 
or engaging in 'spiritual warfare') is actually encouraged (Klassen, 2007) and these groups may likely agree with the notion that the human mind is porous. Relatedly, a limitation of the present study is that all participants were recruited in countries in which the majority religion is Christianity. Thus our scale needs to be further validated in a non-Western context and/or countries with a different majority religion. Based on the theoretical proposal that Eastern compared to Western cultures may be characterized by a porous view of the mind (Taylor, 2007), it could be hypothesized that overall scores on the PToM scale would be higher in Eastern countries and also within folk religions that promote divination (e.g., numerology, tarot etc.).

Our findings are in line with the theoretical notion that religious and spiritual believers are characterized by a different notion of the mind than nonbelievers (Cassaniti \& Luhrmann, 2011): the mind is not seen as bounded but as porous, such that thoughts and spirits can enter or leave the mind. Thereby this study provides an empirical validation of the idea that the concept of 'mind' does not only differ between cultures (i.e. western vs. non-western; cf. Lillard, 1998), but also differs between religious and non-religious people and between people from different religious denominations within the same culture. Similar suggestions for the notion that our view of the mind is strongly culturally determined can be found in studies indicating that intuitions about mind-body dualism (i.e., seeing the body and the mind as two separate entities) differ between cultures and gradually change through a process of socialization (Astuti \& Harris, 2008; Bering \& Bjorklund, 2004).

Following cultural learning accounts of religiosity and paranormal beliefs that have argued for a central role of culture in acquiring supernatural beliefs (Farias et al., 2017; Gervais \& Henrich, 2010; Maij et al., 2017), we suggest that socialization and cultural learning play a strong role in shaping one's (porous) conception of the mind as well. Supernatural beliefs and experiences and the view that the mind is porous are likely mutually reinforcing. For instance, the belief that the spirit of a deceased person still exists and that communication with that spirit is possible implies adopting a porous view of the mind. Beliefs and expectations that the mind is porous and that thoughts can travel - in turn - likely make people more prone to having experiences of 'thought intrusion', 'mindreading' or 'supernatural communication' (for theoretical account of how prior beliefs shape religious experiences, see for instance: Schjoedt et al., 2013; Taves \& Asperem, 2017). Thus, being raised in a religious community will encourage the acquisition of both supernatural beliefs, as well as the view that the mind is a porous entity.

Our studies have implications for theories on the relation between cognitive biases and supernatural beliefs. A basic assumption of theories on so-called 
hyperactive agency detection is that an evolved bias to make false positives and to attribute mental states to non-living objects is at the basis of supernatural beliefs (Barrett, 2000; Boyer, 2003; Guthrie, 1993). Several studies have indeed suggested that supernatural believers are characterized by an enhanced propensity for mentalizing and agency detection (Norenzayan, Gervais, \& Trzesniewski, 2012; Schjoedt, Stdkilde-Jorgensen, Geertz, \& Roepstorff, 20o9; van Elk, 2013; Willard \& Norenzayan, 2013). Our studies suggest that next to being characterized by a hyperactive mentalizing or theory-of-mind system, supernatural believers may actually have a more porous view of the mind than non-believers and atheists. Thereby, this study provides an important contribution to the literature and an interesting question for future studies would be to determine the relative role of hyperactive mentalizing vs. porous mentalizing in explaining supernatural beliefs. In addition, an intriguing possibility would be to explore the potential for experimentally manipulating belief in a porous theory of mind, for instance through the use of thought-transfer or mind-reading paradigms (Swiney \& Sousa, 2013).

Many researchers have focused on how people perceive the mind of God and how this tendency develops across the lifespan (Bering, 2002; Bering \& Johnson, 2005; Gray, Jenkins, Heberlein, \& Wegner, 2011; Gray \& Wegner, 2010; Knight, Sousa, Barrett, \& Atran, 2004; Purzycki et al., 2012; Shtulman, 2008). By using classical theory of mind-reasoning tasks it has been found for instance that children from about 5 years onward come to conceive of God as a different agent than humans (Knight et al., 2004). In addition, it has been found that people attribute more psychological properties than biological or physical properties to God (Shtulman, 2008). It has also been shown that people believe that God has specifically epistemic access to strategic social information, related to cheating and lying for instance (Purzycki et al., 2012). Interestingly, in one study it was found that when believers were encouraged to take God's perspective on a moral issue, they tended to show a reduced bias for non-believing outgroup members (Ginges, Sheikh, Atran, \& Argo, 2016). From an evolutionary psychological perspective, it has been argued that belief in God's privileged epistemic access, functions as an important social monitoring mechanism (Bering \& Johnson, 2005). The continuous feeling that one's thoughts and behavior are being monitored by an omniscient supernatural being may function as a powerful motivator of prosocial behavior (Norenzayan \& Shariff, 2008). Although these different studies have focused on how people perceive the mind of God, little attention has been paid to the implications of this view for how religious believers view the human mind. An interesting avenue for future studies would be to investigate whether one's belief that the mind is porous, is also related to measures of prosocial behavior and ingroup morality. As one example, the 
belief that one's thoughts are transparent for God or supernatural entities may result in more prosocial behavior (e.g. Gervais \& Norenzayan, 2012).

\section{$7 \quad$ Conclusions}

We developed a 4-item porous theory of mind (PToM) scale and confirmed that a one-factor structure best accounted for the variance observed in the data. We established the construct validity of the PToM scale by showing the relation with paranormal beliefs, and by showing that different groups of believers differ in expected ways in their view of the mind as a bounded vs. a porous entity. We propose that our newly developed scale is a parsimonious instrument for measuring, predicting and differentiating paranormal and traditional religious beliefs and experiences in future work/research.

\section{Acknowledgements}

This study was funded by a grant from the Netherlands Organization for Scientific Research (NWO: VENI Grant No. o16.135.135). We thank Marjolein Fokkema for her help with the CFA of Study 4.

\section{References}

Astuti, R., \& Harris, P. L. (2008). Understanding mortality and the life of the ancestors in rural Madagascar. Cognitive Science, 32(4), 713-740. doi:10.1080/03640210802066907.

Barrett, J. L. (2000). Exploring the natural foundations of religion. Trends in Cognitive Sciences, 4(1), 29-34. doi:S1364-6613(99)o1419-9 [pii].

Bering, J. M. (2002). The existential theory of mind. Review of General Psychology, 6(1), 3-24. doi:Doi 10.1037//1089-2680.6.1.3.

Bering, J. M., \& Bjorklund, D. F. (2004). The natural emergence of reasoning about the afterlife as a developmental regularity. Developmental Psychology, 40(2), 217-233. doi:10.1037/0o12-1649.40.20.217.

Bering, J. M., \& Johnson, D. D. (2005). "O Lord ... You Perceive my Thoughts from Afar": Recursiveness and the Evolution of Supernatural Agency. Journal of Cognition and Culture, 5(1), 118-142.

Boyd, A. (1996). Dangerous obsessions: teenagers and the occult. London: Marshall Pickering. 
Boyer, P. (2003). Religious thought and behaviour as by-products of brain function. Trends in Cognitive Sciences, 7(3), 119-124. doi:10.1016/S1364-6613(o3)ooo31-7.

Buhrmester, M., Kwang, T., \& Gosling, S. D. (2011). Amazon's Mechanical Turk: A New Source of Inexpensive, Yet High-Quality, Data? Perspectives on Psychological Science, 6(1), 3-5. doi:10.1177/1745691610393980.

Cassaniti, J. L., \& Luhrmann, T. M. (2011). Encountering the supernatural: A phenomenological account of mind. Religion and Society: Advances in Research, 2(1), 37-53.

Cassaniti, J. L., \& Luhrmann, T. M. (2014). The cultural kindling of spiritual experiences. Current Anthropology, 55(10), 333-343.

Chou, C. P., Bentler, P. M., \& Satorra, A. (1991). Scaled test statistics and robust standard errors for non-normal data in covariance structure analysis: a Monte Carlo study. British Journal of Mathematical and Statistical Psychology, 44, 347-357.

Cohen, A. B., Hall, D. E., Koenig, H. G., \& Meador, K. G. (2005). Social versus individual motivation: Implications for normative definitions of religious orientation. Personality and Social Psychology Review, 9(1), 48-61. doi:Doi 10.1207/ S15327957psprogo1_4.

Emmons, C. F., \& Sobal, J. (1981). Paranormal Beliefs - Functional Alternatives to Mainstream Religion. Review of Religious Research, 22(4), 301-312. doi:Doi $10.2307 / 3509764$.

Enders, C. K. (2001). The impact of nonnormality on full information maximumlikelihood estimation for structural equation models with missing data. Psychological methods, 6(352).

Farias, M., van Mulukom, V., Kahane, G., Kreplin, U., Joyce, A., Soares, P., \& Möttönen, R. (2017). Supernatural belief is not modulated by intuitive thinking style or cognitive inhibition. Scientific Reports.

Fenwick, P., Galliano, S., Coate, M. A., Rippere, V., \& Brown, D. (1985). Psychic Sensitivity, Mystical Experience, Head-Injury and Brain Pathology. British Journal of Medical Psychology, 58(Mar), 35-44.

Field, A. (2009). Discovering statistics using SPSS. London: SAGE Publications Inc.

Gerbing, D. W., \& Hamilton, J. G. (1996). Viability of exploratory factor analysis as a precursor to confirmatory factor analysis. Structural Equation Modeling: A Multidisciplinary Journal, 3(1), 62-72.

Gervais, W. M., \& Henrich, J. (2010). The Zeus problem: Why representational content biases cannot explain faith in gods. Journal of Cognition and Culture, 10 (3), 383-389.

Gervais, W. M., \& Norenzayan, A. (2012). Like a camera in the sky? Thinking about God increases public self-awareness and socially desirable responding. Journal of Experimental Social Psychology, 48(1), 298-302. doi:10.1016/j.jesp.2011.o9.oo6.

Gibbs, R. W., \& Obrien, J. E. (1990). Idioms and Mental-Imagery - the Metaphorical Motivation for Idiomatic Meaning. Cognition, 36(1), 35-68. doi:Doi 10.1016/oo10 -0277(90)90053-M. 
Ginges, J., Sheikh, H., Atran, S., \& Argo, N. (2016). Thinking from God's perspective decreases biased valuation of the life of a nonbeliever. Proceedings of the National Academy of Sciences of the United States of America, 113(2), 316-319. doi:10.1073/ pnas.1512120113.

Glorfeld, L. W. (1995). An improvement on Horn's parallel analysis methodology for selecting the correct number of factors to retain. Educational and psychological measurement, 55(3), 377-393.

Gray, K., Jenkins, A. C., Heberlein, A. S., \& Wegner, D. M. (2011). Distortions of mind perception in psychopathology. Proceedings of the National Academy of Sciences of the United States of America, 108(2), 477-479. doi:Doi 10.1073/Pnas.1015493108.

Gray, K., \& Wegner, D. M. (2010). Blaming God for Our Pain: Human Suffering and the Divine Mind. Personality and Social Psychology Review, 14(1), 7-16. doi: 10.1177/1088868309350299.

Guthrie, S. (1993). Faces in the Clouds: A New Theory of Religion. Oxford: Oxford University Press.

Haslam, N., Kashima, Y., Loughnan, S., Shi, J. Q., \& Suitner, C. (2008). Subhuman, inhuman, and superhuman: Contrasting humans with nonhumans in three cultures. Social Cognition, 26(2), 248-258. doi:Doi 10.1521/Soco.20o8.26.2.248.

Irmak, M. K. (2014). Schizophrenia or Possession? Journal of Religion \& Health, 53(3), 773-777. doi:10.1007/s10943-012-9673-y.

Irwin, H. J. (2009). The psychology of paranormal belief: A researcher's handbook. Hatfield: Univ of Hertfordshire Press.

Jong, J., Bluemke, M., \& Halberstadt, J. (2013). Fear of Death and Supernatural Beliefs: Developing a New Supernatural Belief Scale to Test the Relationship. European Journal of Personality, 27(5), 495-506. doi:Doi 10.1002/Per.1898.

Klassen, P. E. (2007). Radio mind: Protestant experimentalists on the frontiers of healing. Journal of the American Academy of Religion, 75(3), 651-683. doi:10.1093/ jaarel/lfmo4o.

Knight, N., Sousa, P., Barrett, J. L., \& Atran, S. (2004). Children's attributions of beliefs to humans and God: cross-cultural evidence. Cognitive Science, 28(1), 117-126. doi:10.1016/j.cogsci.2003.09.002.

Lakoff, G., \& Johnson, M. (1980). The Metaphorical Structure of the Human Conceptual System. Cognitive Science, 4(2), 195-208. doi:Doi 10.1207/S15516709cogo402_4.

Li, Y. J., Johnson, K. A., Cohen, A. B., Williams, M. J., Knowles, E. D., \& Chen, Z. S. (2012). Fundamental(ist) Attribution Error: Protestants Are Dispositionally Focused. Journal of Personality and Social Psychology, 102(2), 281-29o. doi:Doi 10.1037/Aoo26294.

Lillard, A. (1998). Ethnopsychologies: cultural variations in theories of mind. Psychological Bulletin, 123(1), 3-32.

Lindeman, M., \& Svedholm, A. M. (2012). What's in a Term? Paranormal, Superstitious, Magical and Supernatural Beliefs by Any Other Name Would Mean the Same. Review of General Psychology, 16(3), 241-255. doi:10.1037/aoo27158. 
Luhrmann, T. M. (1991). Persuasions of the witch's craft: ritual magic in contemporary England. Cambridge, MA: Harvard University Press.

Luhrmann, T. M. (2012). When God talks back: Understanding the American evangelical relationship with God. New York: Vintage Books.

Luhrmann, T. M., \& Morgain, R. (2012). Prayer as Inner Sense Cultivation: An Attentional Learning Theory of Spiritual Experience. Ethos, 40(4), 359-389. doi:10.1111/j.1548-1352.2012.01266.x.

Luhrmann, T. M., Nusbaum, H., \& Thisted, R. (2010). The Absorption Hypothesis: Learning to Hear God in Evangelical Christianity. American Anthropologist, 112(1), 66-78. doi:Doi 10.1111/J.1548-1433.2009.01197.X.

Maij, D. L., van Harreveld, F., Gervais, W., Schrag, Y., Mohr, C., \& van Elk, M. (2017). Mentalizing skills do not differentiate believers from non-believers, but credibility enhancing displays do. PLoS One, 12(8), eo182764.

Norenzayan, A., Gervais, W. M., \& Trzesniewski, K. H. (2012). Mentalizing Deficits Constrain Belief in a Personal God. PLoS One, 7(5). doi:ARTN e3688010.1371/journal .pone.oo3688o.

Norenzayan, A., \& Shariff, A. F. (2008). The origin and evolution of religious prosociality. Science, 322(5898), 58-62. doi:10.1126/science.1158757.

Podsakoff, P. M., MacKenzie, S. B., Lee, J. Y., \& Podsakoff, N. P. (2003). Common method biases in behavioral research: A critical review of the literature and recommended remedies. Journal of Applied Psychology, 88(5), 879-903. doi:10.1037/oo21-9101.88.5.879.

Purzycki, B. G., Finkel, D. N., Shaver, J., Wales, N., Cohen, A. B., \& Sosis, R. (2012). What Does God Know? Supernatural Agents' Access to Socially Strategic and Non-Strategic Information. Cognitive Science, 36(5), 846-869. doi:10.1111/j.1551-6709.2012.01242.x.

RC, T. (2014). R: A language and environment for statistical computing. Vienna, Austria: R Foundation for Statistical Computing. Retrieved from http://www.R-project.org/. Rice, T. W. (2003). Believe it or not: Religious and other paranormal beliefs in the United States. Journal for the Scientific Study of Religion, 42(1), 95-106. doi:Doi 10.1111/1468-59o6.00163.

Riekki, T., Lindeman, M., \& Lipsanen, J. (2013). Conceptions about the mind-body problem and their relations to afterlife beliefs, paranormal beliefs, religiosity, and ontological confusions. Advances in Cognitive Psychology, 9(3), 112-120.

Russell, D. W. (2002). In search of underlying dimensions: The use (and abuse) of factor analysis in Personality and Social Psychology Bulletin. Personality and Social Psychology Bulletin, 28(12), 1629-1646. doi:Doi 10.1177/o14616702237645.

Schjoedt, U., Sørensen, J., Nielbo, K. L., Xygalatas, D., Mitkidis, P., \& Bulbulia, J. (2013). Cognitive resource depletion in religious interactions. Religion, Brain \& Behavior, $3(1), 39-55$. 
Schjoedt, U., Stdkilde-Jorgensen, H., Geertz, A. W., \& Roepstorff, A. (2009). Highly religious participants recruit areas of social cognition in personal prayer. Social Cognitive and Affective Neuroscience, 4(2), 199-207. doi:Doi 10.1093/Scan/Nsno5o.

Schmidt, L. E. (2000). Hearing Things: Religion, Illusion and the American Enlightenment. Cambridge, MA: Harvard University Press.

Shtulman, A. (2008). Variation in the anthropomorphization of supernatural beings and its implications for cognitive theories of religion. Journal of Experimental Psychology-Learning Memory and Cognition, 34(5), 1123-1138. doi:10.1037/0278 $-7393 \cdot 34 \cdot 5 \cdot 1123$.

Swiney, L., \& Sousa, P. (2013). When our thoughts are not our own: Investigating agency misattributions using the Mind-to-Mind paradigm. Consciousness and Cognition, 22(2), 589-6o2. doi:10.1016/j.concog.2013.03.007.

Taves, A., \& Asperem, E. (2017). Experience as event: Event cognition and the study of (religious) experience. Religion, Brain \& Behavior, 7(1), 43-62.

Taylor, C. (2007). A Secular Age. Cambridge, MA: Harvard University Press.

Tobacyk, J. (2004). A Revised Paranormal Belief Scale. The International Journal of Transpersonal Studies, 23, 94-98.

van Elk, M. (2013). Paranormal believers are more prone to illusory agency detection than skeptics. Consciousness and Cognition, 22(3), 1041-1046. doi:10.1016/j.concog .2013.07.004 S1053-8100(13)00097-4 [pii].

van Elk, M., Rutjens, B. T., \& van Harreveld, F. (2017). Why Are Protestants More Prosocial Than Catholics? A Comparative Study Among Orthodox Dutch Believers. The International Journal for the Psychology of Religion, 27(1), 65-81.

Willard, A. K., \& Norenzayan, A. (2013). Cognitive biases explain religious belief, paranormal belief, and belief in life's purpose. Cognition, 129(2), 379-391. doi:Doi 10.1016/J.Cognition.2013.07.016.

Winkelman, M. (2002). Shamanism as neurotheology and evolutionary psychology. American BehavioralScientist, 45(12),1875-1887. doi:Doi10.1177/ooo27640212875376o. 